\title{
Weighted Gene Correlation Network Analysis Identifies Specific Functional Modules and Genes in Esophageal Cancer
}

\author{
Wei Xu, Jian Xu, Zhiqiang Wang, and Yuequan Jiang $\mathbb{C}$ \\ Chongqing Key Laboratory of Translational Research for Cancer Metastasis and Individualized Treatment, \\ Chongqing University Cancer Hospital, Chongqing 400030, China
}

Correspondence should be addressed to Yuequan Jiang; jyq1964@yahoo.com

Received 25 November 2021; Revised 12 December 2021; Accepted 14 December 2021; Published 27 December 2021

Academic Editor: Fu Wang

Copyright $(2021$ Wei Xu et al. This is an open access article distributed under the Creative Commons Attribution License, which permits unrestricted use, distribution, and reproduction in any medium, provided the original work is properly cited.

Objective. Esophageal cancer (ESCA) is one of the most aggressive malignancies globally with an undesirable five-year survival rate. Here, this study was conducted for determining specific functional genes linked with ESCA initiation and progression. Methods. Gene expression profiling of ESCA was curated from TCGA (containing 160 ESCA and 11 nontumor specimens) and GSE38129 (30 paired ESCA and nontumor tissues) datasets. Differential expression analysis was conducted between ESCA and nontumor tissues with adjusted $p$ value $<0.05$ and $\mid \log 2$ fold-change $\mid>1$. Weighted gene coexpression network analysis (WGCNA) was conducted for determining the ESCA-specific coexpression modules and genes. Thereafter, ESCA-specific differentially expressed genes (DEGs) were intersected. Functional enrichment analysis was then presented with clusterProfiler package. Protein-protein interaction was conducted, and hub genes were determined. Association of hub genes with pathological staging was evaluated, and survival analysis was presented among ESCA patients. Results. This study determined 91 ESCA-specific DEGs following intersection of DEGs and ESCA-specific genes in TCGA and GSE38129 datasets. They were remarkably linked to cell cycle progression and carcinogenic pathways like the p53 signaling pathway, cellular senescence, and apoptosis. Ten ESCAspecific hub genes were determined, containing ASPM, BUB1B, CCNA2, CDC20, CDK1, DLGAP5, KIF11, KIF20 A, TOP2A, and TPX2. They were prominently associated with pathological staging. Among them, KIF11 upregulation was in relation to undesirable prognosis of ESCA patients. Conclusion. Collectively, we determined ESCA-specific coexpression modules and hub genes, which offered the foundation for future research concerning the mechanistic basis of ESCA.

\section{Introduction}

Esophageal cancer (ESCA) ranks the eighth major cancer type as well as the sixth major cause of cancer-relevant deaths across the globe [1]. Tobacco and alcohol consumption are the main environmental risk factors of ESCA. The five-year survival rate is nearly $15 \%$ [2]. It mainly contains two histological subtypes: esophageal squamous cell carcinoma (approximately 90\%) and esophageal adenocarcinoma (around 10\%) [3]. Patients' advanced clinical presentation is linked to locally late and distant metastasis, which contributes to undesirable survival outcome. Additionally, because of tumor heterogeneity and acquired drug resistance, inherent resistance to radiotherapy and chemotherapy triggers therapeutic failure and unfavorable survival rate [2]. ESCA therapy depends upon patients' and tumors' features, especially the tumor, node, metastasis (TNM) staging system [4]. In the early stage, patients are suitable for endoscopic resection, while those in the advanced stage receive surgical resection, chemotherapy, chemoradiotherapy, or their combination [4]. For patients with unresectable ESCA, systemic chemotherapy is applied. Additionally, immunotherapy has emerged as a therapeutic option for advanced or metastatic patients [5]. Although the therapeutic options have been steadily increasing, the molecular mechanisms underlying ESCA remain indistinct.

The pathogenesis of ESCA is a multistep process, involving distinct stages until eventually cancers [6]. Hence, to focus on the molecular mechanisms underlying the initiation and progression of ESCA may assist uncover underlying diagnostic markers or treatment targets. Weighted gene coexpression network analysis (WGCNA) is a reliable 
systematic biological algorithm, which may emphasize coexpression genomic modules and effectively evaluate the interactions between coexpression modules and clinical phenotypes [7]. This algorithm has been widely utilized for discovering cancer-specific modules and hub genes like bladder cancer [7], hepatocellular carcinoma [8], and lung cancer [9]. Limited studies have applied the WGCNA method to uncover the pathogenesis of ESCA. For instance, Nangraj et al. identified hub genes shared between Barrett's esophagus and esophageal adenocarcinoma through integrated analysis of protein-protein interaction (PPI) and WGCNA [10]. Through WGCNA, $m i R-92 b-3 p$ was determined as a pathogenic gene in ESCA [11]. Integrated analysis of WGCNA and network pharmacology deciphered the molecular mechanisms of compound Kushen injection in ESCA treatment [12]. Here, this study adopted the WGCNA algorithm for determining specific functional modules and genes in ESCA, offering the foundation for future research concerning the mechanistic basis of ESCA.

\section{Materials and Methods}

2.1. Data Collection and Preprocessing. The RNA-seq data of ESCA were retrieved from the Cancer Genome Atlas (TCGA) GDC Application Programming Interface. Gene expression profiling data (read counts) were processed and transformed into gene ID Ensembl (version 90). In total, 160 ESCA and 11 normal tissues were included. Microarray expression profiling of $30 \mathrm{ESCC}$ tumors and adjacent normal tissues was curated from the GSE38129 dataset [13] in the Gene Expression Omnibus (GEO; https://www.ncbi.nlm. nih.gov/gds/) repository. This dataset was in accordance with the GPL571 platform ((HG-U133 A_2) Affymetrix Human Genome U133A 2.0 Array). The raw expression profiling was background-corrected and normalized by quantile utilizing the robust multiarray average (RMA) method.

2.2. Differential Expression Analysis. Differentially expressed genes (DEGs) were selected utilizing the linear models for microarray data (limma; version 3.50.0) package through comparison of the expression profiling between ESCA and normal tissues [14]. The matched $p$ values of gene symbols following the $t$-test were calculated, and adjusted $p$ value $<0.05$ and $\mid \log 2$ fold-change $\mid>1$ were set as the selection criteria. The volcano and heatmap of the DEGs were drawn.

2.3. WGCNA. Coexpression networks were separately established in TCGA and GSE38129 datasets utilizing WGCNA package (version 1.69) [15]. The genes with the first $25 \%$ standard deviation were chosen as the input genes. For constructing a scale-free network, the optimal soft threshold power value ( $\beta$; ranging from 1 to 20 ) was determined with the "pickSoftThreshold" function through calculation of the scale-free fit index. Pearson's correlation matrix was conducted for evaluating the similarity among the pairwise genes utilizing the "cor" function. Thereafter, the adjacency was determined in accordance with $\beta$ and
Pearson's correlation matrix utilizing the "TOMsimilarity" function. Meanwhile, the corresponding dissimilarity (dissTOM) was determined. The modules were segmented with a dynamic cut tree algorithm, and similar modules were merged into one. Module eigengenes (MEs) that were the first principal component of gene expression patterns within a specific module were identified for each module.

2.4. Identification of ESCA-Relevant Coexpression Models. In this study, the most crucial critical feature was tissue type that was designated as ESCA tumor and normal specimens. Pearson correlation between MEs and clinical feature was analyzed. Modules that possessed the strongest correlation coefficient were determined as the ESCA-relevant coexpression models. Module membership indicates the intramodule connectivity of any gene within a given module. The higher the absolute value of module membership, the higher the negative or positive correlation between the gene with the module eigengenes. Gene significance was utilized for incorporating external information to the coexpression network. The higher the absolute value of gene significance, the higher the biological significance of a gene for tissue type. ESCA-relevant genes within the ESCA-relevant coexpression models were determined in accordance with module membership $>0.8$ and gene significance $>0.5$.

2.5. Identification of ESCA-Specific DEGs. For achieving the intersection of DEGs and coexpressed genes, an online web tool (http://bioinfogp.cnb.csic.es/tools/venny/index.html) was adopted for plotting Venn diagram.

2.6. Function Enrichment Analysis. Functional annotation of ESCA-specific DEGs was presented with the clusterProfiler package (version 4.2.0), containing Gene Ontology (GO) and Kyoto Encyclopedia of Genes and Genomes (KEGG) pathway analysis [16]. GO terms comprised of the biological process (BP), cellular component (CC), and molecular function (MF).

2.7. Protein-Protein Interaction (PPI) Analysis. The PPI network of ESCA-specific DEGs was conducted on the basis of the Search Tool for the Retrieval of Interacting Genes/ Proteins (STRING; version 11.0; https://string-db.org) online tool [17]. The CytoHubba plugin [18] of Cytoscape software (version 3.7.2) [19] was adopted for selecting the hub genes within the PPI network [18]. Herein, the first 10 genes were determined as hub genes.

2.8. Survival Analysis. In accordance with the optimal cutoff value determined by survival package, ESCA patients were stratified into high and low expression groups of the 10 ESCA-specific hub genes. Kaplan-Meier curves of overall survival were conducted between groups, and log-rank tests were utilized for comparing the survival differences. 
2.9. Statistical Analysis. All the analyses in this study were implemented utilizing $R$ software (version 3.5.1). Student's $t$ test or Wilcoxon test was adopted for comparisons between groups. Spearman correlation analysis was carried out to evaluate the interactions of the 10 ESCA-specific hub genes with pathological staging of ESCA patients. $P$ value $<0.05$ indicated the statistical significance.

\section{Results}

3.1. Exploration of DEGs in ESCA. For investigating the genetic alterations during the progression from normal to ESCA, we conducted differential expression analysis between ESCA tumors and normal tissues both in TCGA and GSE38129 datasets. In TCGA cohort, compared with 11 normal tissues, 1221 genes presented remarkable downregulation while 1169 genes displayed prominent upregulation in 160 ESCA tumors in accordance with adjusted $p$ value $<0.05$ and $\mid \log 2$ fold-change $\mid>1$ (Figures 1 (a) and $1(\mathrm{~b})$; Supplementary Table 1). With the same selection criteria, in the GSE38129 dataset, we determined 360 upregulated and 376 downregulated genes in 30 ESCA tumors in comparison to 30 nontumor tissues (Figures 1(c) and 1(d); Supplementary Table 2).

\subsection{Establishment of a Coexpression Network and Discovery of} ESCA-Specific Coexpression Module in the TCGA Dataset. We first curated gene expression matrix of ESCA patients from TCGA cohort and chose the genes with the top 25\% variances for subsequent analysis. No outlier sample was found, and we conducted a sample clustering tree, as shown in Figure 2(a). Thereafter, the soft threshold power value was set as 10 (scale-free topology $R^{2}=0.90$ ) for constructing a scale-free network (Figure 2(b)). The adjacency matrix and the topological overlap matrix were separately developed. In total, 9 coexpression modules were clustered in accordance with the average hierarchical clustering and dynamic cutting tree (Figure 2(c)). The association of coexpression modules with clinical trait was analyzed. In Figure 2(d), the yellow module displayed the strongest correlation to tissue type, indicating that this module was strongly linked to ESCA progression. In line with module membership $>0.8$ and gene significance $>0.5$, we determined ESCA-specific genes (Figures 2(e) and 2(f)).

\subsection{Development of a Coexpression Network and Discovery of} ESCA-Specific Coexpression Module in the GSE38129 Cohort. The coexpression network was also developed in the GSE38129 dataset. In accordance with the mRNA expression matrix, we selected the genes with the top 25\% variances. As shown in Figure 3(a), there was no outlier sample among 30 paired ESCA tumors and nontumors. Afterwards, we established a scale-free network in line with the soft threshold power value $=20$ (scale-free topology $R^{2}=0.90$; Figure 3(b)). Following construction of the adjacency matrix and the topological overlap matrix, we determined 7 coexpression modules on the basis of the average hierarchical clustering and dynamic cutting tree
(Figure 3(c)). In Figure 3(d), the turquoise module presented the strongest association with tissue type, demonstrating that this module was strongly linked to ESCA progression. Following module membership $>0.8$ and gene significance $>0.5$, ESCA-specific genes were determined (Figures 3(e) and 3(f)).

\subsection{Identification of ESCA-Specific DEGs and Their Biological} Significance. For determining ESCA-specific DEGs, we intersected the DEGs and the ESCA-specific genes in TCGA and GSE38129 cohorts. As a result, 91 ESCA-specific DEGs were finally identified (Figure 4(a) and Table 1). Their biological significance was further evaluated through GO and KEGG enrichment analysis. In Figure 4(b) and Table 2, we noted that the ESCA-specific DEGs were remarkably linked to cell cycle progression like chromosome segregation, nuclear division, mitotic nuclear division, and sister chromatid segregation. Additionally, the ESCA-specific DEGs were in relation to ESCA progression-relevant KEGG pathways like cell cycle, DNA replication, cellular senescence, base excision repair, mismatch repair, p53 signaling pathway, homologous recombination, nucleotide excision repair, and apoptosis (Figure 4(c) and Table 3).

3.5. Establishment of a PPI Network and Discovery of ESCASpecific Hub Genes. For uncovering the interactions of the ESCA-specific DEGs, we conducted a PPI network in accordance with the STRING online tool. As shown in Figure 5(a), there were close interactions of proteins derived from the ESCA-specific DEGs. Utilizing CytoHubba plugin, we further determined the 10 ESCA-specific hub genes among them, containing TOP2A (score $=4.45 \mathrm{E}+23)$, $A S P M \quad($ score $=4.45 \mathrm{E}+23), \quad C D K 1 \quad($ score $=4.45 \mathrm{E}+23)$, $C D C 20 \quad($ score $=4.45 \mathrm{E}+23)$, CCNA2 $($ score $=4.45 \mathrm{E}+23)$, KIF20 A (score $=4.45 \mathrm{E}+23), K I F 11 \quad($ score $=4.45 \mathrm{E}+23)$, DLGAP5 $($ score $=4.45 \mathrm{E}+23)$, TPX2 $($ score $=4.45 \mathrm{E}+23)$, and $B U B 1 B$ (score $=4.45 \mathrm{E}+23$; Figure 5(b)). These ESCAspecific hub genes might exert crucial roles in ESCA progression.

3.6. Association of the ESCA-Specific Hub Genes with Pathological Staging of ESCA. Further analysis was carried out for evaluating the associations of the ten ESCA-specific hub genes with diverse pathological staging of ESCA patients in TCGA cohort. Our results demonstrated that $A S P M$, $B U B 1 B, C C N A 2$, CDC20, CDK1, DLGAP5, KIF11, KIF20 A, $T O P 2 A$, and TPX2 presented the different expression in diverse pathological stages across ESCA patients (Figures 6(a)-6(j)). This indicated that the 10 ESCA-specific hub genes were remarkably linked to pathological staging of ESCA.

3.7. Association of the ESCA-Specific Hub Genes with ESCA Patients' Prognosis. In accordance with the optimal cutoff value of the expression of the ESCA-specific hub genes, we stratified ESCA patients in TCGA cohort into high and expression groups of $A S P M, B U B 1 B, C C N A 2, C D C 20$, 

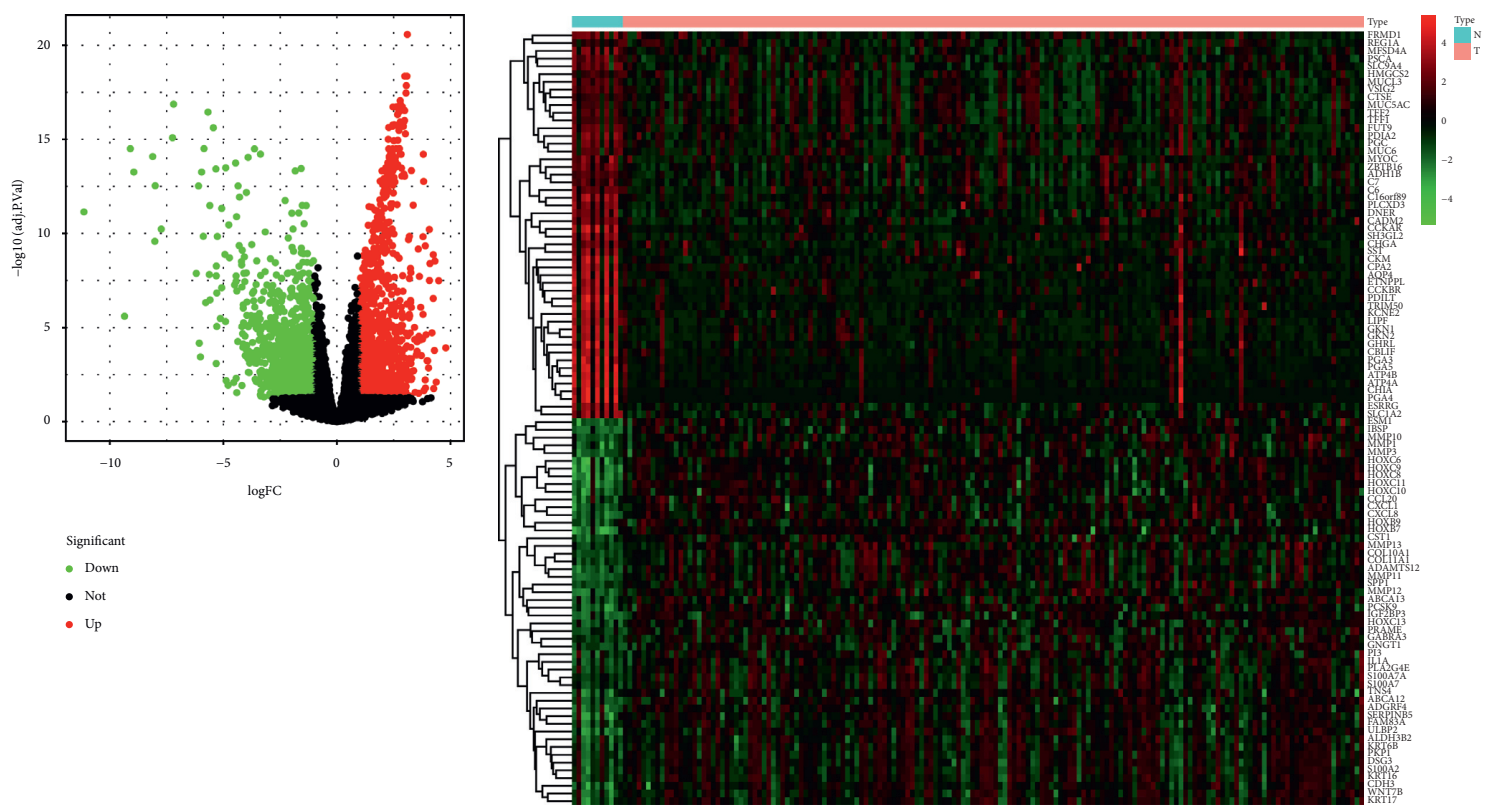

(a)

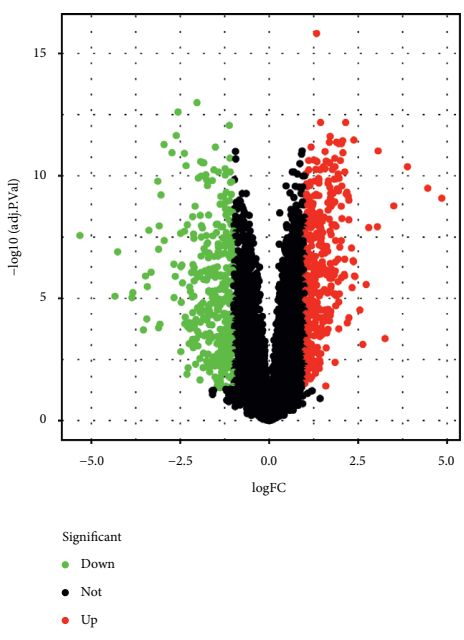

(b)

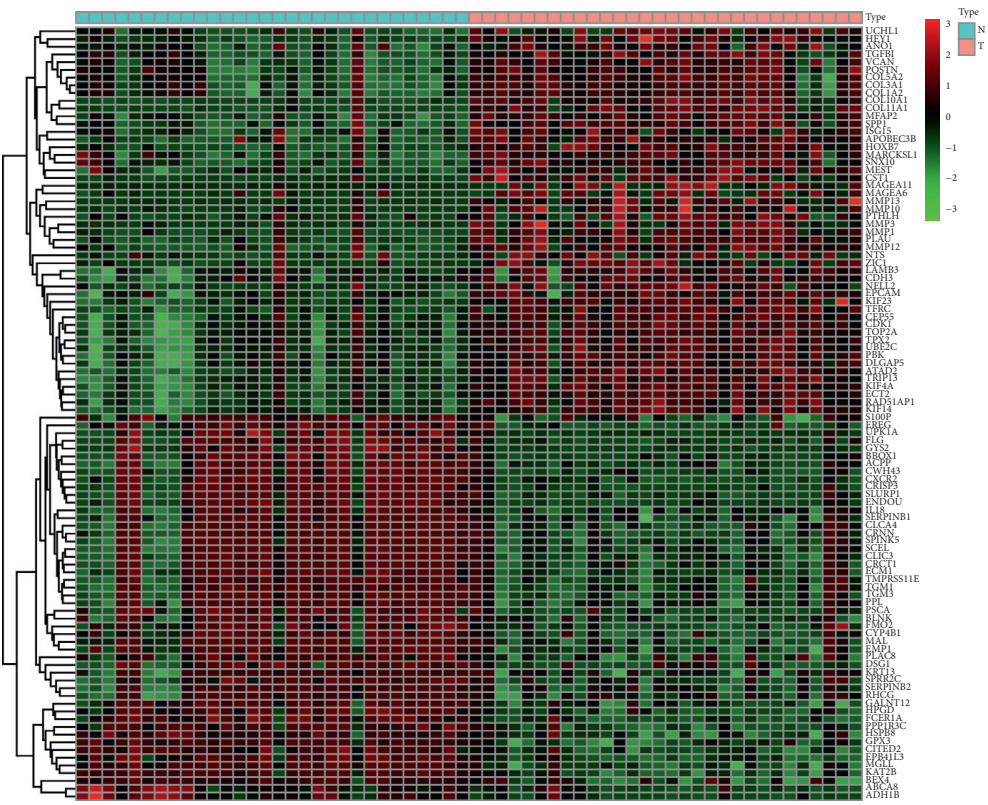

(d)

(c)

FIgURE 1: Analysis of DEGs of ESCA both in TCGA and GSE38129 datasets. (a) Volcano plots depict the results of differential expression analysis between 160 ESCA tumors and 11 normal tissues in TCGA cohort. Red bubble indicates upregulated gene in ESCA; green bubble represents downregulated gene in ESCA; black bubble is indicative of nonsignificant gene. (b) Heatmap visualizes the expression patterns of DEGs with adjusted $p$ value $<0.05$ and $\mid \log 2$ fold-change $\mid>1$ in 160 ESCA tumors (T) and 11 normal tissues (N) in TCGA cohort. Red represents upregulation, while green indicates downregulation. (c) Volcano plots present the results of differential expression analysis between 30 paired ESCA tumors and nontumor tissues in the GSE38129 dataset. Red bubble expresses upregulated gene in ESCA; green bubble is indicative of downregulated gene in ESCA; black bubble represents nonsignificant gene. (d) Heatmap displays the expression patterns of DEGs with adjusted $p$ value $<0.05$ and $\mid \log 2$ fold-change $\mid>1$ in 30 paired ESCA tumors $(\mathrm{T})$ and nontumor tissues $(\mathrm{N})$ in the GSE38129 dataset. Red is indicative of upregulation while green is indicative of downregulation.

CDK1, DLGAP5, KIF11, KIF20A, TOP2A, and TPX2 (Figures $7(\mathrm{a})-7(\mathrm{j}))$. Among them, we noted that ESCA patients in the high expression of the KIF11 group presented more undesirable overall survival outcome in comparison to those in the low expression of the KIF11 group.

\section{Discussion}

High-throughput sequencing technologies have improved our understanding about the heterogeneity and molecular basis underlying ESCA. At present, available biomarkers for 


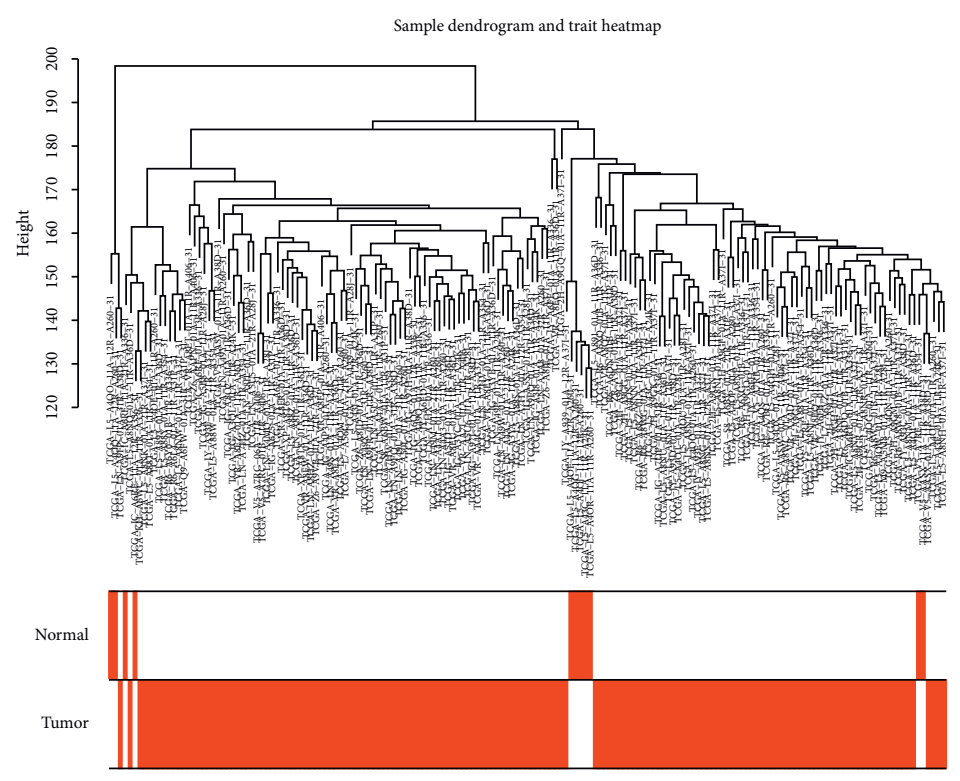

(a)

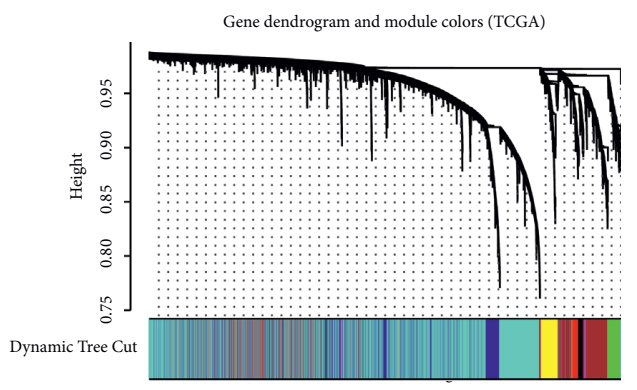

(c)

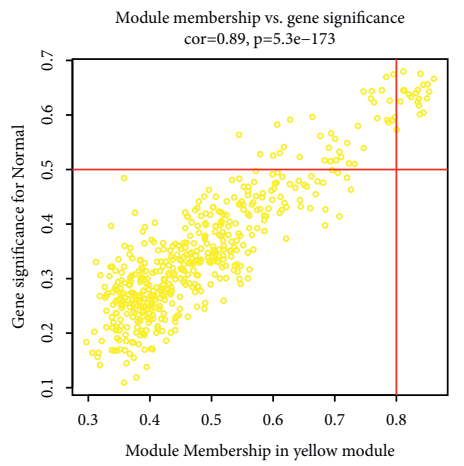

(e)
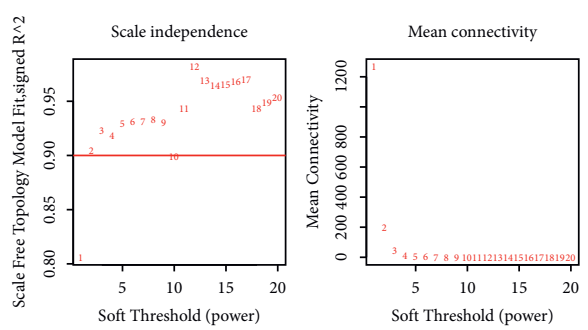

(b)

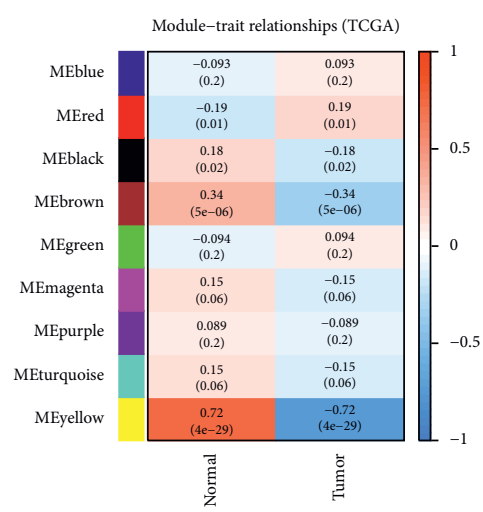

(d)

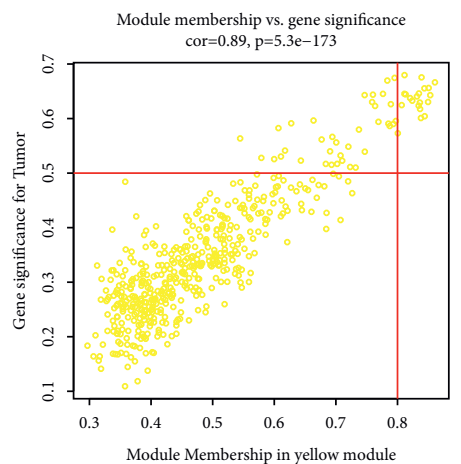

(f)

FIGURE 2: Establishment of a coexpression network and discovery of ESCA-specific coexpression module in the TCGA dataset. (a) Sample cluster analysis. (b) The scale-free network topology (left) as well as mean connectivity (right) under distinct soft threshold power values. (c) Gene dendrogram clustered in accordance with a dissimilarity measure. The upper panel indicates gene tree, and the bottom panel represents gene modules identified by diverse colors. (d) Heatmap visualizes the interaction between coexpression modules and clinical trait-tissue type. The upper number in each cell presents Pearson correlation coefficient between each module and tissue type. Meanwhile, the lower number indicates the $p$ value. (e) Scatter plots depict the interaction between module membership and gene significance for normal tissue type for the yellow module. (f) Scatter plots present the interaction between module membership and gene significance for ESCA tumor tissue type for the yellow module. 

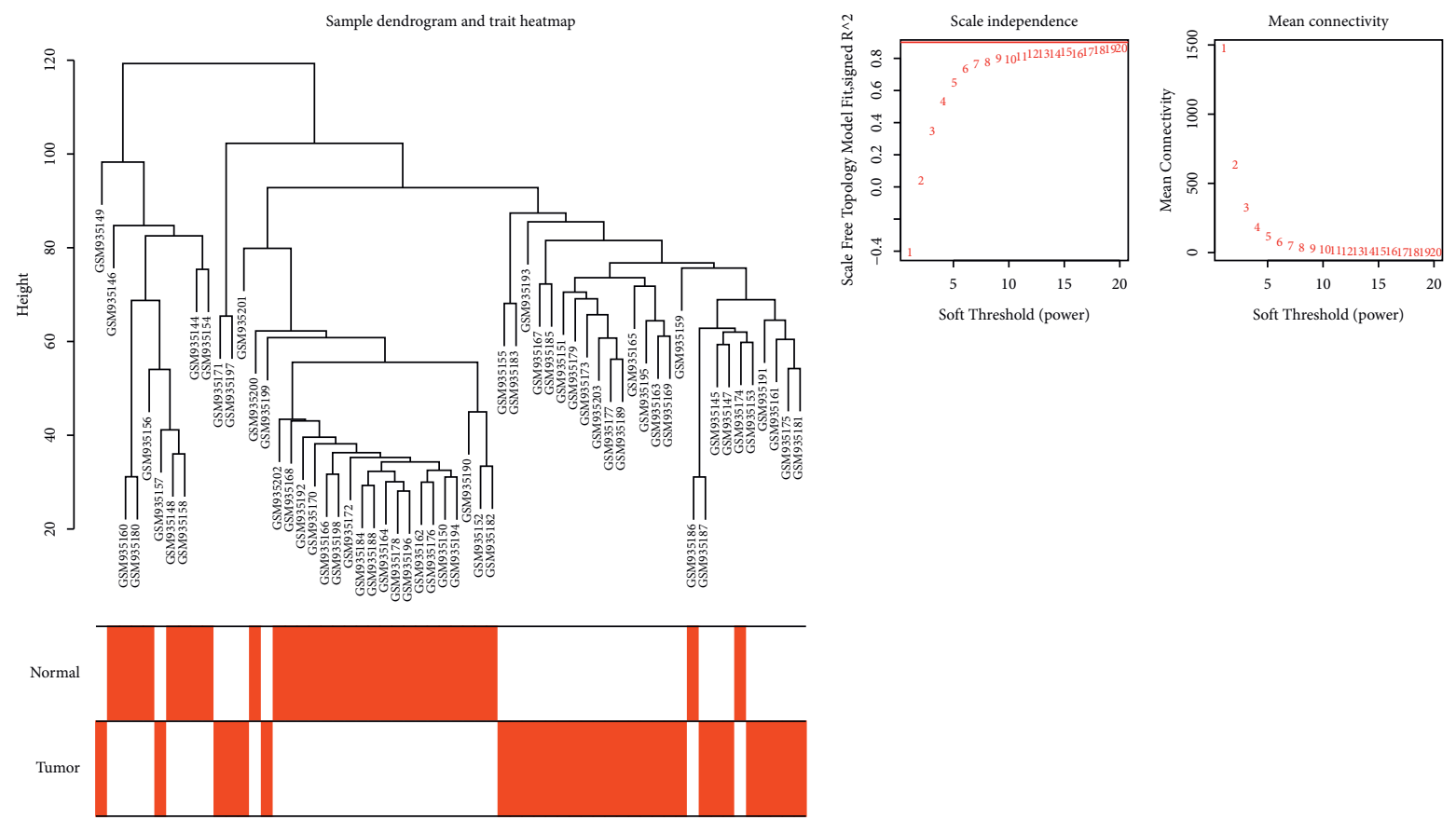

(a)

(b)
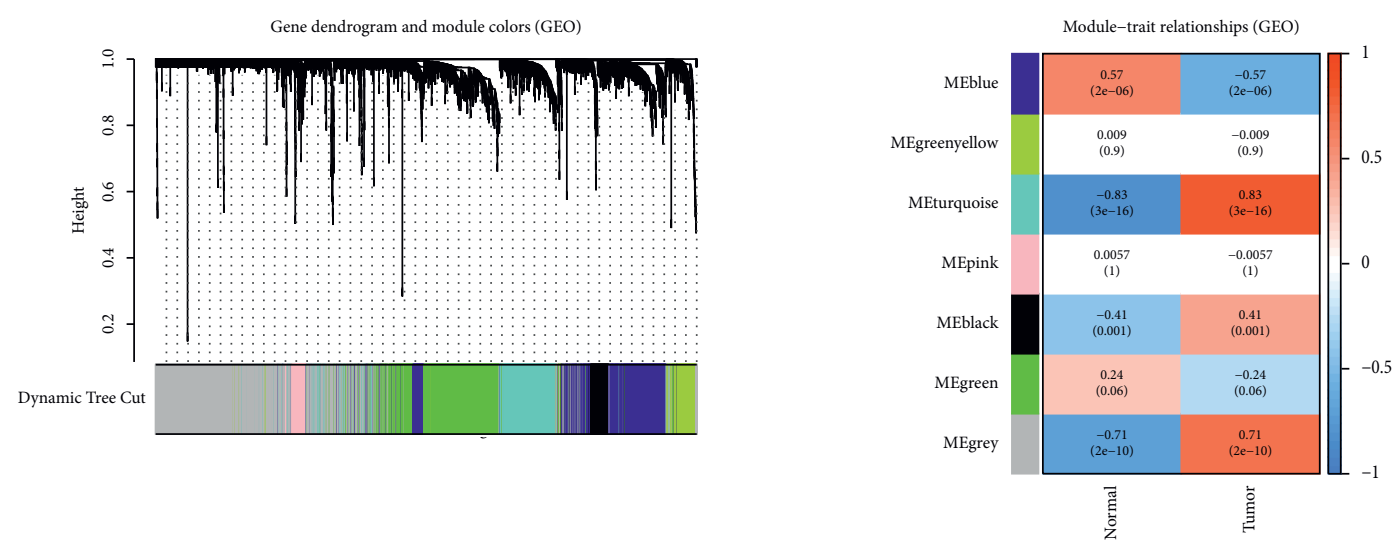

(c)

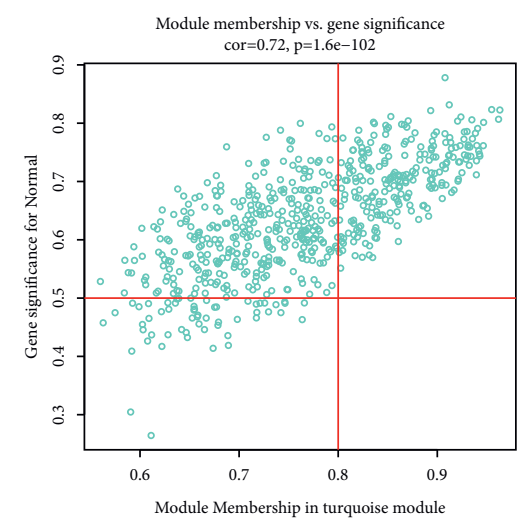

(e) (d)

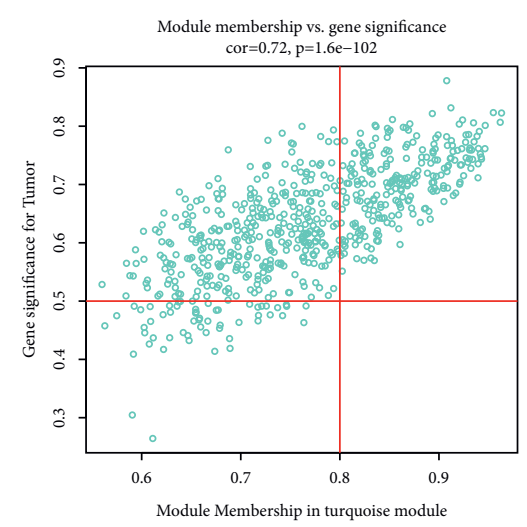

(f)

Figure 3: Development of a coexpression network and discovery of ESCA-specific coexpression module in the GSE38129 cohort. (a) Sample cluster analysis of 30 paired ESCA tumors and nontumors. (b) The scale-free network topology (left) and mean connectivity (right) following diverse soft threshold power values. (c) Gene dendrogram clustered in line with a dissimilarity measure. The upper panel presents gene tree and the bottom panel is indicative of gene modules signed by diverse colors. (d) Heatmap displays the relationship between coexpression modules and clinical trait-tissue type. The upper number in each cell presents Pearson correlation coefficient between each module and tissue type. Additionally, the lower number represents the $p$ value. (e) Scatter plots showing the association between module membership and gene significance for normal tissue type for the turquoise module. (f) Scatter plots present the correlation between module membership and gene significance for ESCA tumor tissue type for the turquoise module. 

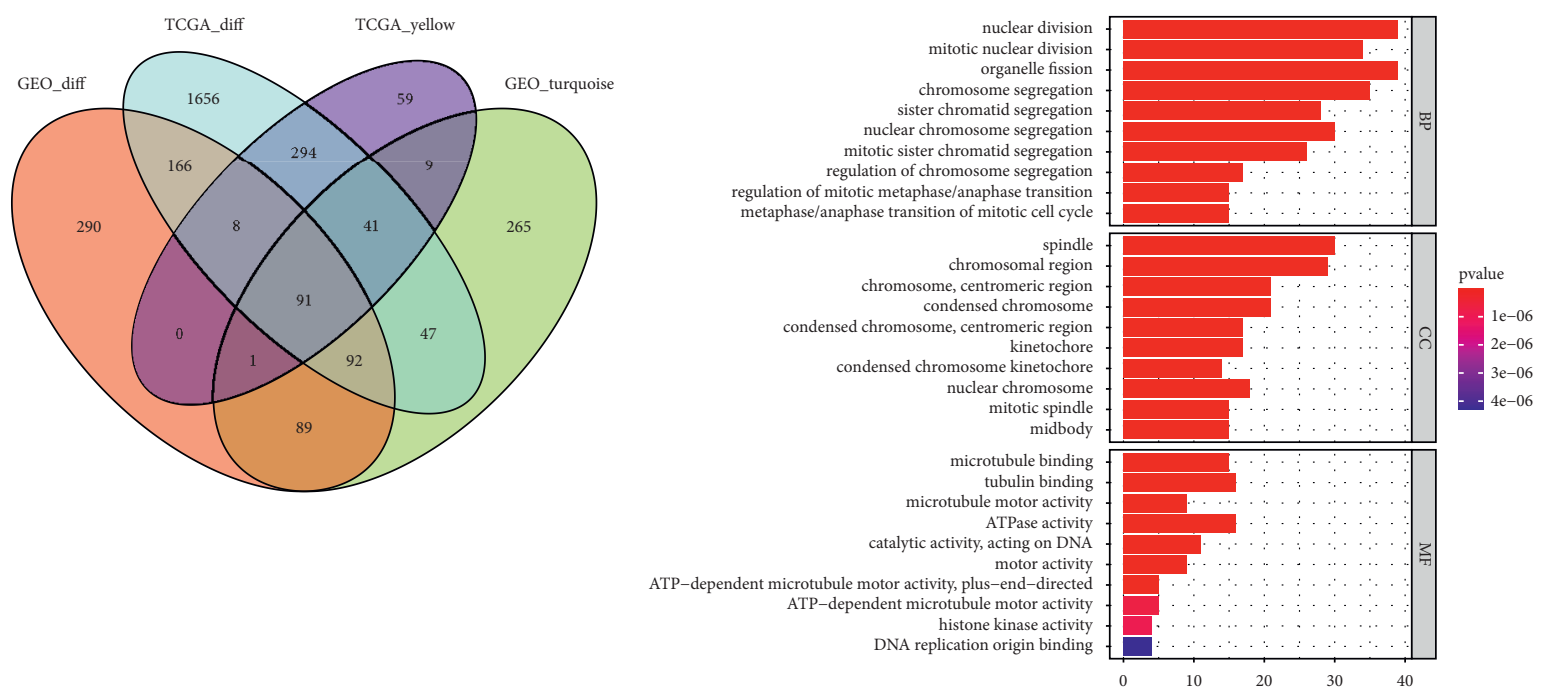

(a)

(b)

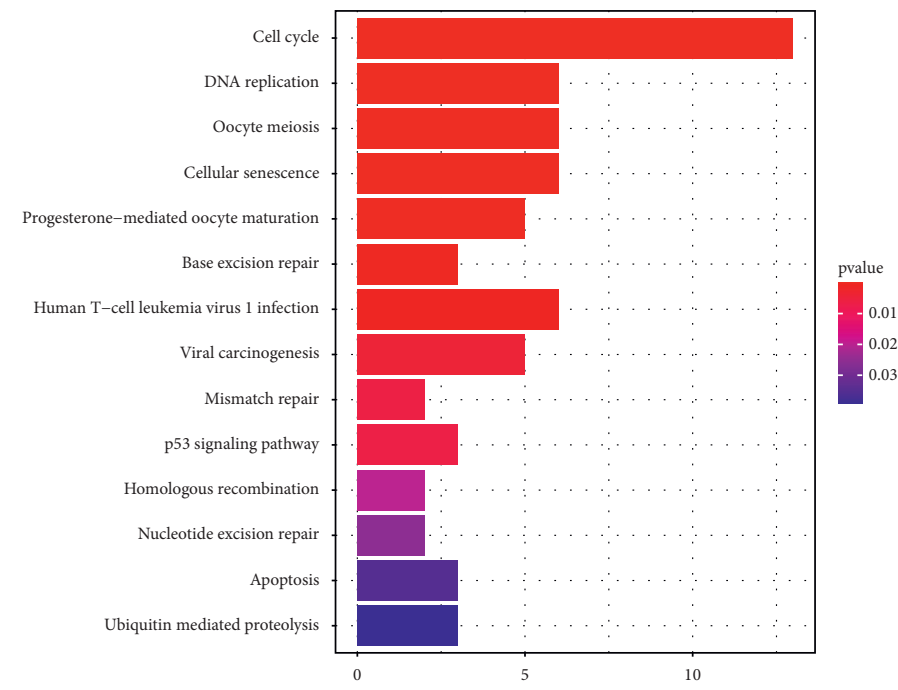

(c)

FIGURE 4: Identification of ESCA-specific DEGs and their biological significance. (a) Venn diagram depicts the intersection of the DEGs and the ESCA-specific genes in TCGA and GSE38129 cohorts. (b) GO enrichment results of the ESCA-specific DEGs. The first 10 enrichment results of BP, CC, and MF categories are separately displayed. (c) KEGG pathway enrichment results of the ESCA-specific DEGs.

prediction of ESCA patients' survival outcome remain nonsufficiently sensitive and specific. Hence, this study was conducted for discovering novel biomarkers for efficiently predicting ESCA patients' prognosis through the WGCNA algorithm, eventually lowering patients' morbidity and mortality.

Combining the DEGs and ESCA-specific genes in TCGA and GSE38129 cohorts, we determined 91 ESCA-specific DEGs. Our functional enrichment analyses uncovered that the ESCA-specific DEGs were remarkably linked to cell cycle progression and carcinogenic pathways like the p53 signaling pathway, cellular senescence, and apoptosis. This indicated that the ESCA-specific DEGs exerted crucial roles in ESCA progression. Additionally, there were prominent interactions between proteins derived from the ESCAspecific DEGs in accordance with the PPI network. Among them, the 10 ESCA-specific hub genes were finally determined, containing ASPM, BUB1B, CCNA2, CDC20, CDK1, DLGAP5, KIF11, KIF20A, TOP2A, and TPX2.

The tumorigenic roles of $A S P M$ have been proposed in diverse cancer types. For instance, $A S P M$ triggers prostate carcinoma stemness and progression through enhancing the Wnt-Dvl-3-beta-catenin pathway [20]. It is predictive of undesirable prognosis and modulates cellular proliferation in bladder carcinoma [21]. Its upregulation accelerates glioblastoma growth through modulating G1 restriction point progression as well as the Wnt-beta-catenin pathway [22]. Aberrantly expressed ASPM regulated by transcriptional factor FoxM1 triggers the malignant progression of gliomas [23]. Additionally, it is linked to poor survival outcome as well as induces carcinogenesis in diffuse large B cell lymphoma [24]. Abnormally expressed ASPM induces the progression of lung squamous cell carcinoma through modulating CDK4 [25]. Increasing evidences demonstrate 
Table 1: The list of ESCA-specific DEGs.

\begin{tabular}{|c|c|c|c|c|}
\hline \multicolumn{5}{|c|}{ ESCA-specific DEGs } \\
\hline CBX3 & FOXM1 & DBF4 & LMNB2 & BLM \\
\hline KAT2B & DLGAP5 & MCM10 & ASPM & C1orf112 \\
\hline KIF4A & PCNA & NUSAP1 & UBE2S & AURKB \\
\hline AURKA & KIF18B & CDT1 & POLE2 & FBXO5 \\
\hline CKS1B & CENPE & BUB1B & OIP5 & MYBL2 \\
\hline ECT2 & CDC6 & BIRC5 & CCNA2 & CHEK1 \\
\hline HOXB7 & CEP55 & NCAPH & CCNB1 & TFRC \\
\hline KIF14 & MCM6 & DTL & MKI67 & UBE2C \\
\hline TRIP13 & PRC1 & NCAPG & DEPDC1 & CDKN3 \\
\hline CITED2 & CDCA3 & FAM189A2 & LMNB1 & KIF20 A \\
\hline MCM2 & FEN1 & HJURP & $\mathrm{NDC1}$ & GINS2 \\
\hline WDHD1 & NUDT1 & ORC6 & KIF11 & CENPF \\
\hline RAD51AP1 & RNASEH $2 A$ & HMMR & GINS1 & STMN1 \\
\hline MAD2L1 & PBK & ECHDC2 & RUVBL1 & EXO1 \\
\hline NDC80 & CKS2 & FYCO1 & CENPM & DNMT1 \\
\hline NEK2 & KIF18A & DDX39A & KNTC1 & CDK1 \\
\hline KIF2C & SECISBP2L & MELK & CDC20 & TPX2 \\
\hline KIF23 & RAD54L & SHCBP1 & TK1 & TOP2A \\
\hline SPC25 & & & & \\
\hline
\end{tabular}

TABLE 2: The detailed information of GO enrichment results of ESCA-specific genes.

\begin{tabular}{|c|c|c|c|c|c|c|c|}
\hline ID & Description & $\begin{array}{l}\text { Gene } \\
\text { ratio }\end{array}$ & BgRatio & $P$ value & Adjusted $p$ & $Q$ value & Count \\
\hline $\begin{array}{l}\text { GO: } \\
0000280\end{array}$ & Nuclear division & $39 / 90$ & $\begin{array}{c}436 / \\
18862\end{array}$ & $4.56 E-40$ & $6.17 E-37$ & $4.56 E-37$ & 39 \\
\hline $\begin{array}{l}\text { GO: } \\
0140014\end{array}$ & Mitotic nuclear division & $34 / 90$ & $\begin{array}{c}296 / \\
18862\end{array}$ & $2.13 E-38$ & $1.44 E-35$ & $1.07 E-35$ & 34 \\
\hline $\begin{array}{l}\text { GO: } \\
0048285\end{array}$ & Organelle fission & $39 / 90$ & $\begin{array}{c}486 / \\
18862\end{array}$ & $3.31 E-38$ & $1.49 E-35$ & $1.10 E-35$ & 39 \\
\hline $\begin{array}{l}\text { GO: } \\
0007059\end{array}$ & Chromosome segregation & $35 / 90$ & $\begin{array}{c}337 / \\
18862\end{array}$ & $5.24 E-38$ & $1.77 E-35$ & $1.31 E-35$ & 35 \\
\hline $\begin{array}{l}\text { GO: } \\
0000819\end{array}$ & Sister chromatid segregation & $28 / 90$ & $\begin{array}{c}199 / \\
18862\end{array}$ & $5.58 E-34$ & $1.51 E-31$ & $1.12 E-31$ & 28 \\
\hline $\begin{array}{l}\text { GO: } \\
0098813\end{array}$ & Nuclear chromosome segregation & $30 / 90$ & $\begin{array}{c}273 / \\
18862\end{array}$ & $4.07 E-33$ & $9.16 E-31$ & $6.77 E-31$ & 30 \\
\hline $\begin{array}{l}\text { GO: } \\
0000070\end{array}$ & Mitotic sister chromatid segregation & $26 / 90$ & $\begin{array}{c}164 / \\
18862\end{array}$ & $6.09 E-33$ & $1.18 E-30$ & $8.70 E-31$ & 26 \\
\hline $\begin{array}{l}\text { GO: } \\
0051983\end{array}$ & Regulation of chromosome segregation & $17 / 90$ & $89 / 18862$ & $4.02 E-23$ & $6.80 E-21$ & $5.02 E-21$ & 17 \\
\hline $\begin{array}{l}\text { GO: } \\
0030071\end{array}$ & Regulation of mitotic metaphase/anaphase transition & $15 / 90$ & $59 / 18862$ & $1.50 E-22$ & $2.25 E-20$ & $1.66 E-20$ & 15 \\
\hline $\begin{array}{l}\text { GO: } \\
0007091\end{array}$ & Metaphase/anaphase transition of mitotic cell cycle & $15 / 90$ & $61 / 18862$ & $2.63 E-22$ & $3.55 E-20$ & $2.63 E-20$ & 15 \\
\hline $\begin{array}{l}\text { GO: } \\
0005819\end{array}$ & Spindle & $30 / 90$ & $\begin{array}{c}381 / \\
19520\end{array}$ & $3.83 E-29$ & $4.34 E-27$ & $2.77 E-27$ & 30 \\
\hline $\begin{array}{l}\text { GO: } \\
0098687\end{array}$ & Chromosomal region & $29 / 90$ & $\begin{array}{c}345 / \\
19520\end{array}$ & $5.72 E-29$ & $4.34 E-27$ & $2.77 E-27$ & 29 \\
\hline $\begin{array}{l}\text { GO: } \\
0000775\end{array}$ & Chromosome, centromeric region & $21 / 90$ & $\begin{array}{c}196 / \\
19520\end{array}$ & $3.40 E-23$ & $1.72 E-21$ & $1.10 E-21$ & 21 \\
\hline $\begin{array}{l}\text { GO: } \\
0000793\end{array}$ & Condensed chromosome & $21 / 90$ & $\begin{array}{c}217 / \\
19520\end{array}$ & $3.00 E-22$ & $1.14 E-20$ & $7.26 E-21$ & 21 \\
\hline $\begin{array}{l}\text { GO: } \\
0000779\end{array}$ & Condensed chromosome, centromeric region & $17 / 90$ & $\begin{array}{c}117 / \\
19520\end{array}$ & $3.24 E-21$ & $9.84 E-20$ & $6.27 E-20$ & 17 \\
\hline $\begin{array}{l}\text { GO: } \\
0000776\end{array}$ & Kinetochore & $17 / 90$ & $\begin{array}{c}137 / \\
19520\end{array}$ & $5.31 E-20$ & $1.35 E-18$ & $8.57 E-19$ & 17 \\
\hline $\begin{array}{l}\text { GO: } \\
0000777\end{array}$ & Condensed chromosome kinetochore & $14 / 90$ & $\begin{array}{c}106 / \\
19520\end{array}$ & $5.13 E-17$ & $1.11 E-15$ & $7.10 E-16$ & 14 \\
\hline $\begin{array}{l}\text { GO: } \\
0000228\end{array}$ & Nuclear chromosome & $18 / 90$ & $\begin{array}{c}250 / \\
19520\end{array}$ & $7.75 E-17$ & $1.47 E-15$ & $9.38 E-16$ & 18 \\
\hline
\end{tabular}


TABLE 2: Continued.

\begin{tabular}{|c|c|c|c|c|c|c|c|}
\hline ID & Description & $\begin{array}{l}\text { Gene } \\
\text { ratio }\end{array}$ & BgRatio & $P$ value & Adjusted $p$ & $Q$ value & Count \\
\hline $\begin{array}{l}\text { GO: } \\
0072686\end{array}$ & Mitotic spindle & $15 / 90$ & $\begin{array}{c}157 / \\
19520\end{array}$ & $5.26 E-16$ & $8.89 E-15$ & $5.67 E-15$ & 15 \\
\hline $\begin{array}{l}\text { GO: } \\
0030496\end{array}$ & Midbody & $15 / 90$ & $\begin{array}{c}193 / \\
19520\end{array}$ & $1.17 E-14$ & $1.77 E-13$ & $1.13 E-13$ & 15 \\
\hline $\begin{array}{l}\text { GO: } \\
0008017\end{array}$ & Microtubule binding & $15 / 89$ & $\begin{array}{c}269 / \\
18337\end{array}$ & $3.07 E-12$ & $5.56 E-10$ & $3.91 E-10$ & 15 \\
\hline $\begin{array}{l}\text { GO: } \\
0015631\end{array}$ & Tubulin binding & $16 / 89$ & $\begin{array}{c}368 / \\
18337\end{array}$ & $2.33 E-11$ & $2.11 E-09$ & $1.49 E-09$ & 16 \\
\hline $\begin{array}{l}\text { GO: } \\
0003777\end{array}$ & Microtubule motor activity & $9 / 89$ & $69 / 18337$ & $4.41 E-11$ & $2.66 E-09$ & $1.87 E-09$ & 9 \\
\hline $\begin{array}{l}\text { GO: } \\
0016887\end{array}$ & ATPase activity & $16 / 89$ & $\begin{array}{c}478 / \\
18337\end{array}$ & $1.09 E-09$ & $4.92 E-08$ & $3.46 E-08$ & 16 \\
\hline $\begin{array}{l}\text { GO: } \\
0140097\end{array}$ & Catalytic activity, acting on DNA & $11 / 89$ & $\begin{array}{c}204 / \\
18337\end{array}$ & $4.22 E-09$ & $1.53 E-07$ & $1.08 E-07$ & 11 \\
\hline $\begin{array}{l}\text { GO: } \\
0003774\end{array}$ & Motor activity & $9 / 89$ & $\begin{array}{c}129 / \\
18337\end{array}$ & $1.26 E-08$ & $3.67 E-07$ & $2.59 E-07$ & 9 \\
\hline $\begin{array}{l}\text { GO: } \\
0008574\end{array}$ & $\begin{array}{l}\text { ATP-dependent microtubule motor activity, plus-end- } \\
\text { directed }\end{array}$ & $5 / 89$ & $17 / 18337$ & $1.42 E-08$ & $3.67 E-07$ & $2.59 E-07$ & 5 \\
\hline $\begin{array}{l}\text { GO: } \\
1990939\end{array}$ & ATP-dependent microtubule motor activity & $5 / 89$ & $35 / 18337$ & $6.96 E-07$ & $1.57 E-05$ & $1.11 E-05$ & 5 \\
\hline $\begin{array}{l}\text { GO: } \\
0035173\end{array}$ & Histone kinase activity & $4 / 89$ & $16 / 18337$ & $9.02 E-07$ & $1.81 E-05$ & $1.28 E-05$ & 4 \\
\hline $\begin{array}{l}\text { GO: } \\
0003688\end{array}$ & DNA replication origin binding & $4 / 89$ & $23 / 18337$ & $4.28 E-06$ & $7.06 E-05$ & $4.97 E-05$ & 4 \\
\hline
\end{tabular}

TABLE 3: The detailed information of KEGG pathways enriched by ESCA-specific genes.

\begin{tabular}{|c|c|c|c|c|c|c|c|}
\hline ID & Description & Gene ratio & BgRatio & $P$ value & Adjusted $p$ & $Q$ value & Count \\
\hline hsa04110 & Cell cycle & $13 / 43$ & $126 / 8104$ & $4.08 E-14$ & $2.08 E-12$ & $1.59 E-12$ & 13 \\
\hline hsa03030 & DNA replication & $6 / 43$ & $36 / 8104$ & $2.69 E-08$ & $6.85 E-07$ & $5.23 E-07$ & 6 \\
\hline hsa04114 & Oocyte meiosis & $6 / 43$ & $131 / 8104$ & $5.94 E-05$ & 0.001009 & 0.000771 & 6 \\
\hline hsa04218 & Cellular senescence & $6 / 43$ & $156 / 8104$ & 0.000156 & 0.001923 & 0.001468 & 6 \\
\hline hsa04914 & Progesterone-mediated oocyte maturation & $5 / 43$ & $102 / 8104$ & 0.000189 & 0.001923 & 0.001468 & 5 \\
\hline hsa03410 & Base excision repair & $3 / 43$ & $33 / 8104$ & 0.00068 & 0.005776 & 0.004411 & 3 \\
\hline hsa05166 & Human $\mathrm{T}$ cell leukemia virus 1 infection & $6 / 43$ & $222 / 8104$ & 0.001031 & 0.007513 & 0.005737 & 6 \\
\hline hsa05203 & Viral carcinogenesis & $5 / 43$ & $204 / 8104$ & 0.004257 & 0.02714 & 0.020726 & 5 \\
\hline hsa03430 & Mismatch repair & $2 / 43$ & $23 / 8104$ & 0.006483 & 0.034097 & 0.026039 & 2 \\
\hline hsa04115 & p53 signaling pathway & $3 / 43$ & $73 / 8104$ & 0.006686 & 0.034097 & 0.026039 & 3 \\
\hline hsa03440 & Homologous recombination & $2 / 43$ & $41 / 8104$ & 0.019784 & 0.091726 & 0.070049 & 2 \\
\hline hsa03420 & Nucleotide excision repair & $2 / 43$ & $47 / 8104$ & 0.025565 & 0.108651 & 0.082974 & 2 \\
\hline hsa04210 & Apoptosis & $3 / 43$ & $136 / 8104$ & 0.035027 & 0.137412 & 0.104938 & 3 \\
\hline hsa04120 & Ubiquitin mediated proteolysis & $3 / 43$ & $142 / 8104$ & 0.039049 & 0.14225 & 0.108633 & 3 \\
\hline
\end{tabular}

the crucial role of $A S P M$ in cancer progression. For example, $B U B 1 B$ accelerates prostate carcinoma proliferation through transcriptionally modulating $M E L K$ [26]. It triggers hepatocellular carcinoma development through activating $m$ TORC1 signaling [27]. It can facilitate extrahepatic cholangiocarcinoma development through $J \mathrm{NK} / \mathrm{c}$-Jun signaling [28]. Moreover, it participates in the tumorigenicity and radioresistance of glioblastoma [29]. For CCNA2, it can be suppressed by $m i R-219-5 \mathrm{p}$, thereby affecting cellular proliferation and cell cycle progression in ESCA [30]. A previous study has proposed that CDC20 modulates E2F1 degradation and thymidylate synthase expression, thereby triggering ESCA chemoresistance [31]. Furthermore, CDK1 has been considered as an underlying diagnostic and cancer progression biomarker as well as a drug target for ESCA [32]. Previous bioinformatics and experimental evidences have demonstrated the tumorigenic role of DLGAP5 in ESCA [33]. KIF11 is essential for spheroid formation of ESCA cells [34]. ScRNA-seq and qPCR analysis uncovered that KIF20A possesses the potential to diagnose and predict ESCA patients' prognosis [35]. For TOP2A, experimental data demonstrate that it can affect the resistance of ESCA cells to paclitaxel [36]. Targeting TPX2 relieves ESCA progression through weakening tumor growth and invasion [37, 38]. Additionally, its upregulation is mediated by LINC00337 and triggers autophagy and resistance to cisplatin in ESCA cells [39]. On the basis of previously published literature and our findings, $A S P M, B U B 1 B, C C N A 2, C D C 20, C D K 1$, 


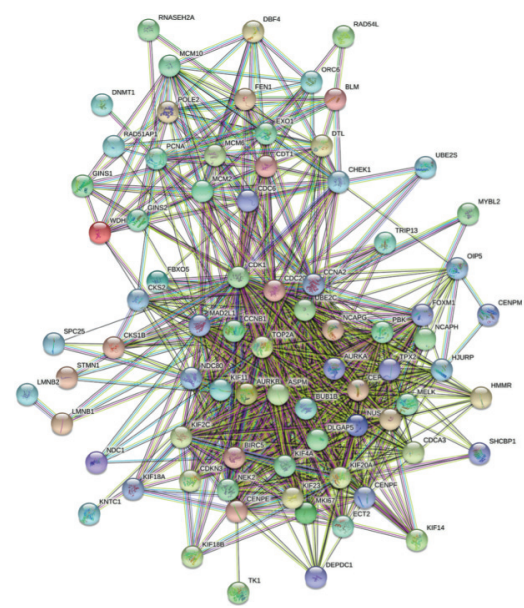

(a)

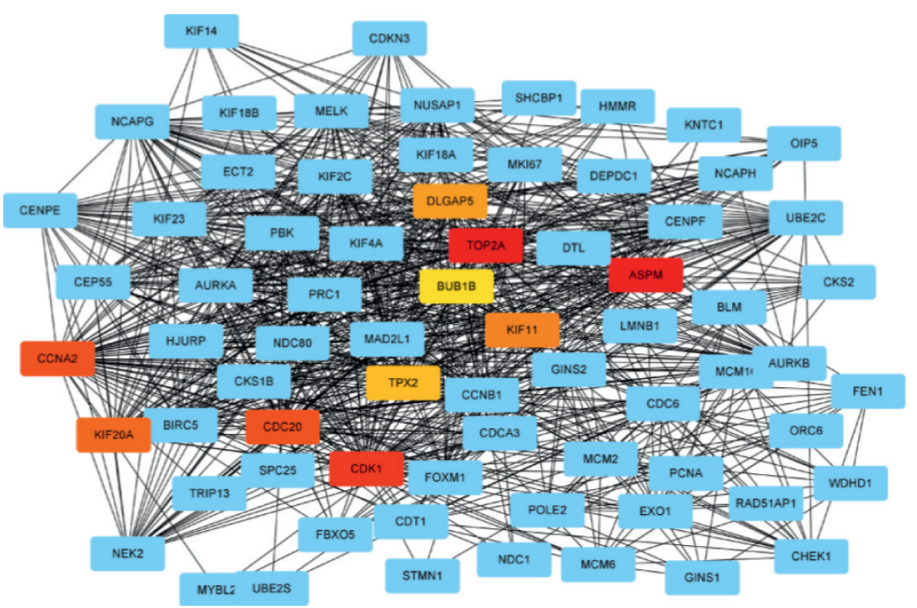

(b)

FIGURE 5: Establishment of a PPI network and discovery of ESCA-specific hub genes. (a) The PPI network of ESCA-specific DEGs through the STRING online tool. (b) Discovery of the ESCA-specific hub genes utilizing CytoHubba plugin. The ten hub genes are marked in orange.

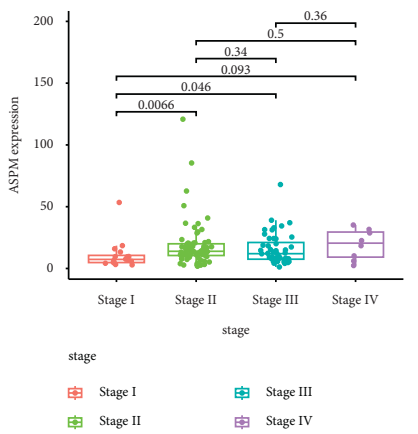

(a)

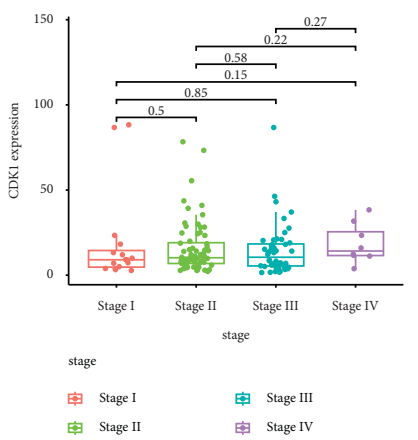

(e)

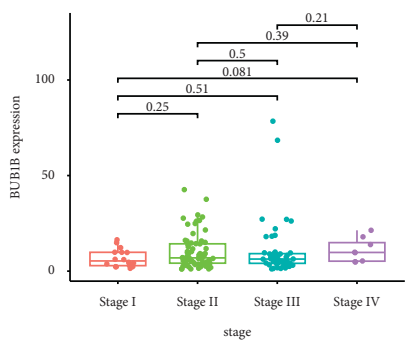

stage

审 Stage I

审 Stage 1

(b)

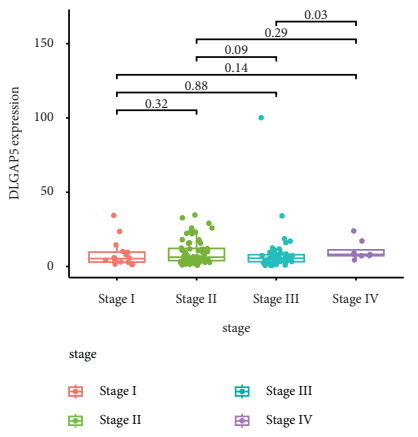

(f)

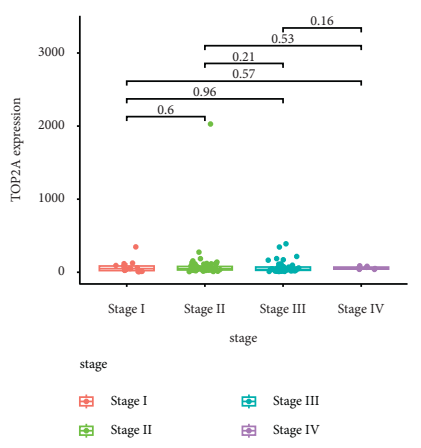

(i)

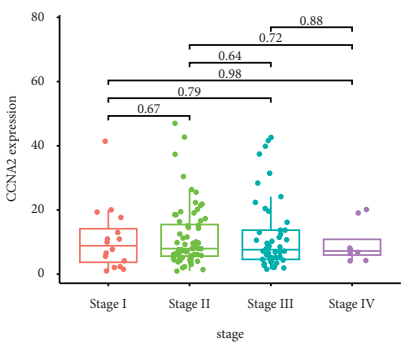

stage

由 Stage I

由 Stage II

由 Stage III

由 Stage IV

(c)

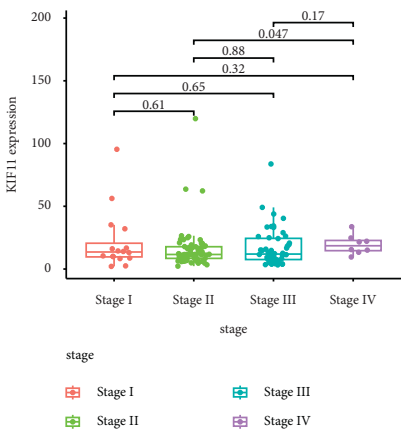

(g)

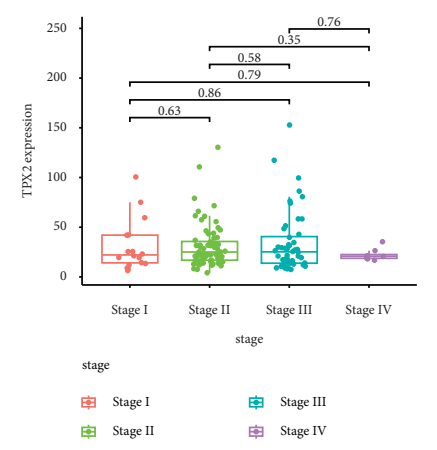

(j)

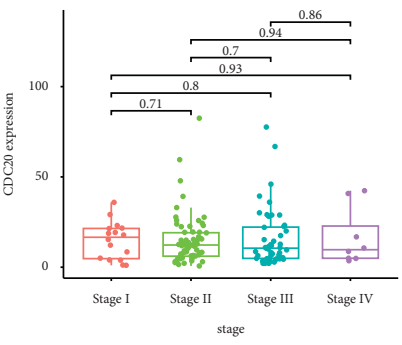

stage

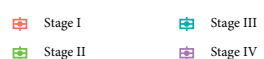

(d)

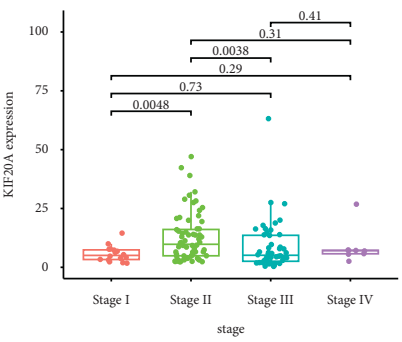

stage

困 Stage I Stage III

审 Stage II 审 Stage IV

(h)

FIGURE 6: Association of the ESCA-specific hub genes with pathological staging of ESCA patients. (a-j) Box plots depict the difference in (a) $A S P M$, (b) BUB1B, (c) CCNA2, (d) CDC20, (e) CDK1, (f) DLGAP5, (g) KIF11, (h) KIF20A, (i) TOP2A, and (j) TPX2 among diverse pathological staging of ESCA patients in TCGA cohort. 


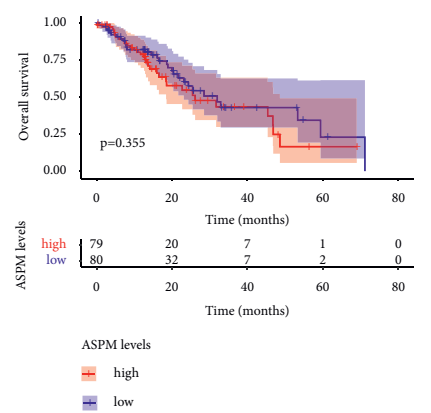

(a)

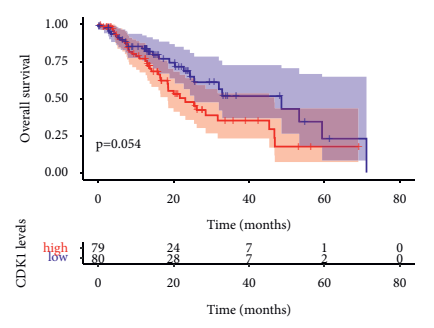

CDK1 levels
$+\quad$ high + high
+ low

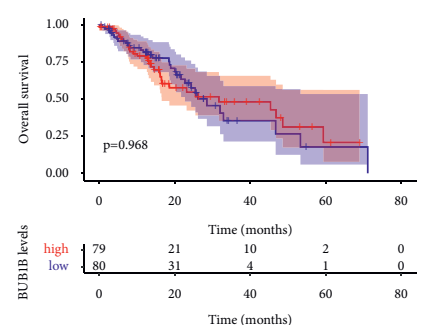

BUBIB levels \pm high + low

(b)

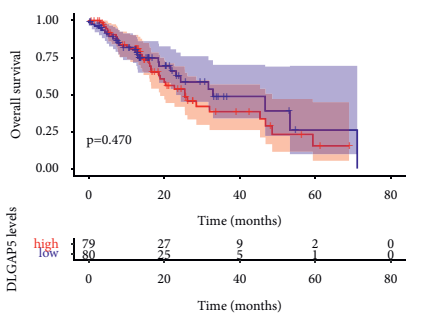

DLGAP5 levels

\pm high

+ low
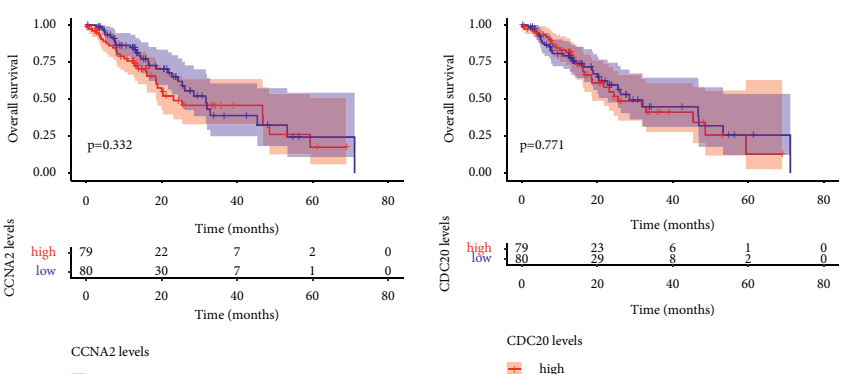

= high

\pm low

(c)
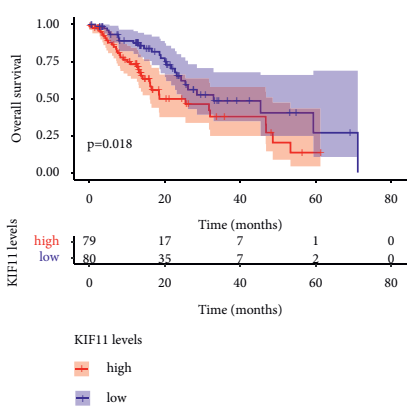

(g)

(h)

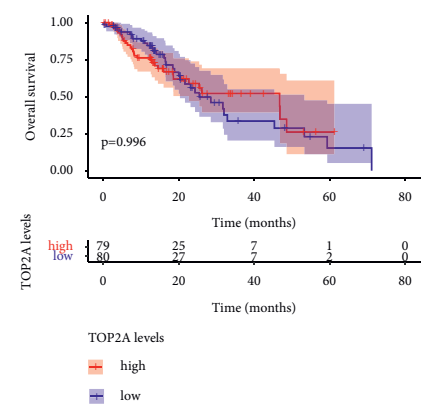

(i)

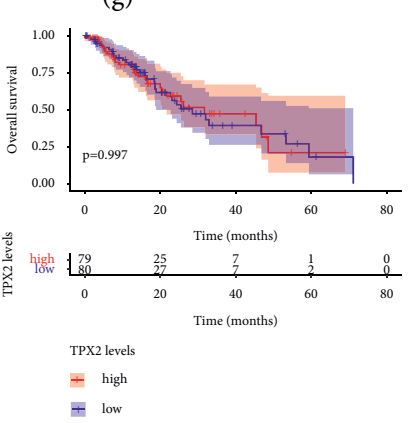

(j)

Figure 7: Association of the ESCA-specific hub genes with ESCA patients' prognosis in TCGA cohort. (a-j) Kaplan-Meier curves display the difference in overall survival between high and low expression of (a) ASPM, (b) BUB1B, (c) CCNA2, (d) CDC20, (e) CDK1, (f) DLGAP5, (g) KIF11, (h) KIF20A, (i) TOP2A, and (j) TPX2 groups. Survival difference between groups is determined with the log-rank test.

DLGAP5, KIF11, KIF20A, TOP2A, and TPX2 play crucial roles in ESCA progression.

Currently, approach of predicting ESCA patients' prognosis primarily depends on the conventional TNM staging system. Although conventional TNM staging is crucial for diagnosis and treatment interventions, it cannot roundly uncover the intrinsic biological processes and pathological development due to the high heterogeneity in tumor microenvironment and individual discrepancy. Our results demonstrated that the 10 ESCA-specific hub genes (ASPM, BUB1B, CCNA2, CDC20, CDK1, DLGAP5, KIF11, $K I F 20 A, T O P 2 A$, and TPX2) presented the remarkable associations with pathological staging, indicating that their roles in ESCA progression. Among them, KIF11 upregulation was indicative of an unfavorable survival outcome of ESCA patients, indicative of the potential of KIF11 as a prognostic indicator of ESCA.

However, there are certain drawbacks in our study. First, the influence of expression alteration of the ESCA-specific hub genes upon patients' prognosis remains to be explored. Hence, in our future, the interactions of the ESCA-specific hub genes with patients' prognosis will be monitored and verified in the large-scale clinical data. Additionally, it is of importance to consider statistical bias because the sample size is relatively small. Moreover, in-depth investigation will be presented for validating the biological significance of the ESCA-specific hub genes through in vitro and in vivo experiments.

\section{Conclusion}

Overall, this study determined the 10 ESCA-specific hub genes as novel markers for ESCA with the WGCNA algorithm based on distinct datasets, which offered promising targets for ESCA precision medicine. Nevertheless, in-depth exploration is required for validating the biological function of the specific hub genes in large-scale clinical cohorts. 


\section{Abbreviations}

ESCA: Esophageal cancer

TNM: $\quad$ Tumor, node, metastasis

WGCNA: Weighted gene coexpression network analysis

TCGA: The Cancer Genome Atlas

GEO: $\quad$ Gene Expression Omnibus

DEGs: Differentially expressed genes

GO: $\quad$ Gene Ontology

KEGG: Kyoto Encyclopedia of Genes and Genomes

BP: Biological process

CC: $\quad$ Cellular component

MF: $\quad$ Molecular function

PPI: $\quad$ Protein-protein interaction

STRING: Search Tool for the Retrieval of Interacting Genes/Proteins.

\section{Data Availability}

The data used to support the findings of this study are included within the supplementary information files.

\section{Conflicts of Interest}

The authors declare that they have no conflicts of interest.

\section{Authors' Contributions}

Wei $\mathrm{Xu}$ and Jian $\mathrm{Xu}$ contributed equally to this work.

\section{Acknowledgments}

This work was funded by Chongqing Medical Scientific Research Project (Joint Project of Chongqing Health Commission and Science and Technology Bureau) (2021MSXM287) and The Planning Decision Consultation Project of Shapingba Science and Technology Bureau (Jcd202018).

\section{Supplementary Materials}

Supplementary Table 1. The detailed information of DEGs between ESCA tumors and normal tissues in TCGA cohort. Supplementary Table 2. The detailed information of DEGs between ESCA tumors and normal tissues in the GSE38129 dataset. . (Supplementary Materials)

\section{References}

[1] F. Bray, J. Ferlay, I. Soerjomataram, R. L. Siegel, L. A. Torre, and A. Jemal, "Global cancer statistics 2018: GLOBOCAN estimates of incidence and mortality worldwide for 36 cancers in 185 countries," CA: A Cancer Journal for Clinicians, vol. 68, no. 6, pp. 394-424, 2018.

[2] Z. Yuan, X. Wang, X. Geng et al., "Liquid biopsy for esophageal cancer: is detection of circulating cell-free DNA as a biomarker feasible?" Cancer Communications, vol. 41, no. 1, pp. 3-15, 2021.

[3] A. A. Bhat, S. Nisar, S. Maacha et al., "Cytokine-chemokine network driven metastasis in esophageal cancer; promising avenue for targeted therapy," Molecular Cancer, vol. 20, no. 1, p. 2, 2021.

[4] W. M. Grady, M. Yu, and S. D. Markowitz, "Epigenetic alterations in the gastrointestinal tract: current and emerging use for biomarkers of cancer," Gastroenterology, vol. 160, no. 3, pp. 690-709, 2021.

[5] Y. Kakeji, T. Oshikiri, G. Takiguchi et al., "Multimodality approaches to control esophageal cancer: development of chemoradiotherapy, chemotherapy, and immunotherapy," Esophagus, vol. 18, no. 1, pp. 25-32, 2021.

[6] W. Li, J. Liu, and H. Zhao, "Identification of a nomogram based on long non-coding RNA to improve prognosis prediction of esophageal squamous cell carcinoma," Aging, vol. 12, no. 2, pp. 1512-1526, 2020.

[7] Z. Tian, W. He, J. Tang et al., "Identification of important modules and biomarkers in breast cancer based on WGCNA," OncoTargets and Therapy, vol. 13, pp. 6805-6817, 2020.

[8] K. H. Bai, S. Y. He, L. L. Shu et al., "Identification of cancer stem cell characteristics in liver hepatocellular carcinoma by WGCNA analysis of transcriptome stemness index," Cancer Medicine, vol. 9, no. 12, pp. 4290-4298, 2020.

[9] M. Ding, F. Li, B. Wang, G. Chi, and H. Liu, "A comprehensive analysis of WGCNA and serum metabolomics manifests the lung cancer-associated disordered glucose metabolism," Journal of Cellular Biochemistry, vol. 120, no. 6, pp. 10855-10863, 2019.

[10] A. S. Nangraj, G. Selvaraj, S. Kaliamurthi, A. C. Kaushik, W. C. Cho, and D. Q. Wei, "Integrated PPI- and WGCNAretrieval of hub gene signatures shared between Barrett's esophagus and esophageal adenocarcinoma," Frontiers in Pharmacology, vol. 11, p. 881, 2020.

[11] W. Wang, S. Fu, X. Lin et al., "miR-92b-3p functions as A key gene in esophageal squamous cell cancer as determined by Co-expression analysis," OncoTargets and Therapy, vol. 12, pp. 8339-8353, 2019.

[12] W. Zhou, J. Wu, J. Zhang et al., "Integrated bioinformatics analysis to decipher molecular mechanism of compound Kushen injection for esophageal cancer by combining WGCNA with network pharmacology," Scientific Reports, vol. 10, no. 1, Article ID 12745, 2020.

[13] N. Hu, C. Wang, R. J. Clifford et al., "Integrative genomics analysis of genes with biallelic loss and its relation to the expression of mRNA and micro-RNA in esophageal squamous cell carcinoma," BMC Genomics, vol. 16, no. 1, p. 732, 2015.

[14] M. E. Ritchie, B. Phipson, D. Wu et al., "Limma powers differential expression analyses for RNA-sequencing and microarray studies," Nucleic Acids Research, vol. 43, no. 7, p. e47, 2015.

[15] B. Zhang and S. Horvath, "A general framework for weighted gene co-expression network analysis," Statistical Applications in Genetics and Molecular Biology, vol. 4, Article ID 17, 2005.

[16] G. Yu, L.-G. Wang, Y. Han, and Q.-Y. He, "clusterProfiler: an $\mathrm{R}$ package for comparing biological themes among gene clusters," OMICS: A Journal of Integrative Biology, vol. 16, no. 5, pp. 284-287, 2012.

[17] D. Szklarczyk, J. H. Morris, H. Cook et al., "The STRING database in 2017: quality-controlled protein-protein association networks, made broadly accessible," Nucleic Acids Research, vol. 45, no. D1, pp. D362-d368, 2017.

[18] C. H. Chin, S. H. Chen, and H. H. Wu, "CytoHubba: identifying hub objects and sub-networks from complex interactome," BMC Systems Biology, vol. 8, no. Suppl 4, p. S11, 2014. 
[19] P. Shannon, A. Markiel, O. Ozier et al., "Cytoscape: a software environment for integrated models of biomolecular interaction networks," Genome Research, vol. 13, no. 11, pp. 2498-2504, 2003.

[20] V. C. Pai, C.-C. Hsu, T.-S. Chan et al., "ASPM promotes prostate cancer stemness and progression by augmenting Wnt-Dvl-3- $\beta$-catenin signaling," Oncogene, vol. 38, no. 8, pp. 1340-1353, 2019.

[21] Z. Y. Gao, F. Yu, H. X. Jia, Z. Ye, and S. J. Yao, “ASPM predicts poor prognosis and regulates cell proliferation in bladder cancer," The Kaohsiung Journal of Medical Sciences, vol. 36, no. 12, pp. 1021-1029, 2020.

[22] X. Chen, L. Huang, Y. Yang et al., "ASPM promotes glioblastoma growth by regulating G1 restriction point progression and Wnt- $\beta$-catenin signaling," Aging, vol. 12, no. 1, pp. 224-241, 2020.

[23] W. J. Zeng, Q. Cheng, Z. P. Wen et al., “Aberrant ASPM expression mediated by transcriptional regulation of FoxM1 promotes the progression of gliomas," Journal of Cellular and Molecular Medicine, vol. 24, no. 17, pp. 9613-9626, 2020.

[24] J. Wu, Z. He, Y. Zhu, C. Jiang, Y. Deng, and B. Wei, “ASPM predicts poor clinical outcome and promotes tumorigenesis for diffuse large B-cell lymphoma," Current Cancer Drug Targets, vol. 21, no. 1, pp. 80-89, 2021.

[25] Y.-J. Yuan, Y. Sun, R. Gao, Z.-z. Yin, Z.-y. Yuan, and L.-M. Xu, "Abnormal spindle-like microcephaly-associated protein (ASPM) contributes to the progression of Lung Squamous Cell Carcinoma (LSCC) by regulating CDK4," Journal of Cancer, vol. 11, no. 18, pp. 5413-5423, 2020.

[26] J.-H. Tian, L.-J. Mu, M.-Y. Wang et al., "BUB1B promotes proliferation of prostate cancer via transcriptional regulation of MELK," Anti-Cancer Agents in Medicinal Chemistry, vol. 20, no. 9, pp. 1140-1146, 2020.

[27] J. Qiu, S. Zhang, P. Wang et al., "BUB1B promotes hepatocellular carcinoma progression via activation of the mTORC1 signaling pathway," Cancer Medicine, vol. 9, no. 21, pp. 8159-8172, 2020.

[28] C. Y. Jiao, Q. C. Feng, C. X. Li et al., "BUB1B promotes extrahepatic cholangiocarcinoma progression via JNK/c-Jun pathways," Cell Death \& Disease, vol. 12, no. 1, p. 63, 2021.

[29] Q. Ma, Y. Liu, L. Shang, J. Yu, and Q Qu, "The FOXM1/ BUB1B signaling pathway is essential for the tumorigenicity and radioresistance of glioblastoma," Oncology Reports, vol. 38, pp. 3367-3375, 2017.

[30] Q. Ma, "MiR-219-5p suppresses cell proliferation and cell cycle progression in esophageal squamous cell carcinoma by targeting CCNA2," Cellular and Molecular Biology Letters, vol. 24, no. 1, p. 4, 2019.

[31] B. Li, W. W. Xu, X. Y. Guan et al., "Competitive binding between Id1 and E2F1 to Cdc20 regulates E2F1 degradation and thymidylate synthase expression to promote esophageal cancer chemoresistance," Clinical Cancer Research, vol. 22, no. 5, pp. 1243-1255, 2016.

[32] D. E. Hansel, S. Dhara, R. C. Huang et al., "CDC2/CDK1 expression in esophageal adenocarcinoma and precursor lesions serves as a diagnostic and cancer progression marker and potential novel drug target," The American Journal of Surgical Pathology, vol. 29, no. 3, pp. 390-399, 2005.

[33] J. Hu, R. Li, H. Miao, and Z. Wen, "Identification of key genes for esophageal squamous cell carcinoma via integrated bioinformatics analysis and experimental confirmation," Journal of Thoracic Disease, vol. 12, no. 6, pp. 3188-3199, 2020.
[34] T. Imai, N. Oue, K. Sentani et al., "KIF11 is required for spheroid formation by oesophageal and colorectal cancer cells," Anticancer Research, vol. 37, no. 1, pp. 47-56, 2017.

[35] L. Zheng, L. Li, J. Xie, H. Jin, and N. Zhu, "Six novel biomarkers for diagnosis and prognosis of esophageal squamous cell carcinoma: validated by scRNA-seq and qPCR," Journal of Cancer, vol. 12, no. 3, pp. 899-911, 2021.

[36] S. Zhang, H. Jiang, Z. Xu et al., "The resistance of esophageal cancer cells to paclitaxel can be reduced by the knockdown of long noncoding RNA DDX11-AS1 through TAF1/TOP2A inhibition," American journal of cancer research, vol. 9, pp. 2233-2248, 2019.

[37] Y. Hou, H. Liu, and W. Pan, "Knockdown of circ_0003340 induces cell apoptosis, inhibits invasion and proliferation through miR-564/TPX2 in esophageal cancer cells," Experimental Cell Research, vol. 394, no. 2, Article ID 112142, 2020.

[38] H.-C. Liu, G.-H. Zhang, Y.-H. Liu et al., "TPX2 siRNA regulates growth and invasion of esophageal cancer cells," Biomedicine \& Pharmacotherapy, vol. 68, no. 7, pp. 833-839, 2014.

[39] C. Yang, S. Shen, X. Zheng et al., "Long non-coding RNA LINC00337 induces autophagy and chemoresistance to cisplatin in esophageal squamous cell carcinoma cells via upregulation of TPX2 by recruiting E2F4," The FASEB Journal, vol. 34, no. 5, pp. 6055-6069, 2020. 